\title{
Correction
}

\section{Correction to: Glycosylation of dentin matrix protein 1 is a novel key element for astrocyte maturation and BBB integrity}

\author{
Bo Jing ${ }^{1}$, Chunxue Zhang ${ }^{1}$, Xianjun Liu ${ }^{1}$, Liqiang Zhou ${ }^{1}$, Jiping Liu ${ }^{1}$, Yinan Yao ${ }^{1}$, Juehua Yu ${ }^{1}$, \\ Yuteng Weng ${ }^{2}$, Min Pan ${ }^{2}$, Jie Liu', Zuolin Wang ${ }^{2 \bowtie}$, Yao Sun ${ }^{2 \bowtie}$, Yi Eve Sun ${ }^{1,3,4 \bowtie}$ \\ 1 Tongji University School of Medicine, Stem Cell Translational Research Center, Tongji Hospital, Shanghai 200065, China \\ 2 Department of Oral Implantology, School of Stomatology, Tongji University, Shanghai Engineering Research Center of Tooth \\ Restoration and Regeneration, Shanghai 200072, China \\ ${ }^{3}$ Department of Psychiatry and Biobehavioral Sciences, David Geffen School of Medicine, University of California, Los \\ Angeles, CA 90095, USA \\ ${ }^{4}$ Collaborative Innovation Center for Brain Science, Tongji University, Shanghai 200092, China \\ $\bowtie$ Correspondence: zuolin@tongji.edu.cn (Z. Wang), yaosun@tongji.edu.cn (Y. Sun), yi.eve.sun@gmail.com (Y. E. Sun)
}

CORRECTION TO: PROTEIN CELL 2018, 9(3):298-309

HTTPS://DOI.ORG/10.1007/S13238-017-0449-8

In the original publication, the label of Fig. $2 \mathrm{C}$ should be read as "GFAP/lectin/DAPI" not "DMP1/GFAP/lectin/DAPI". 
A
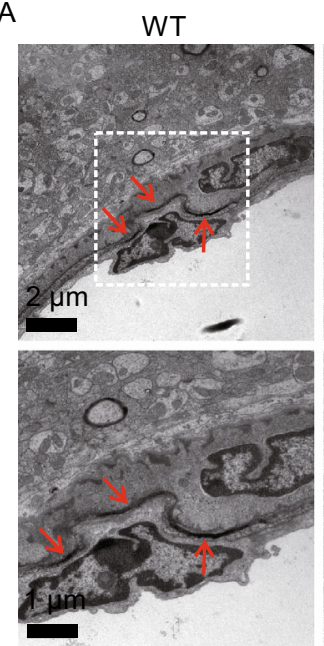

C
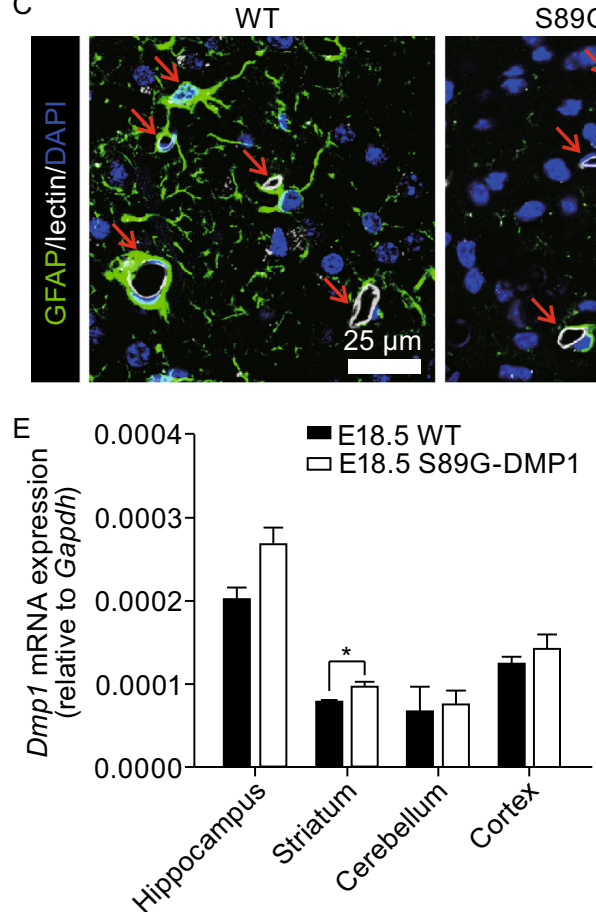

B
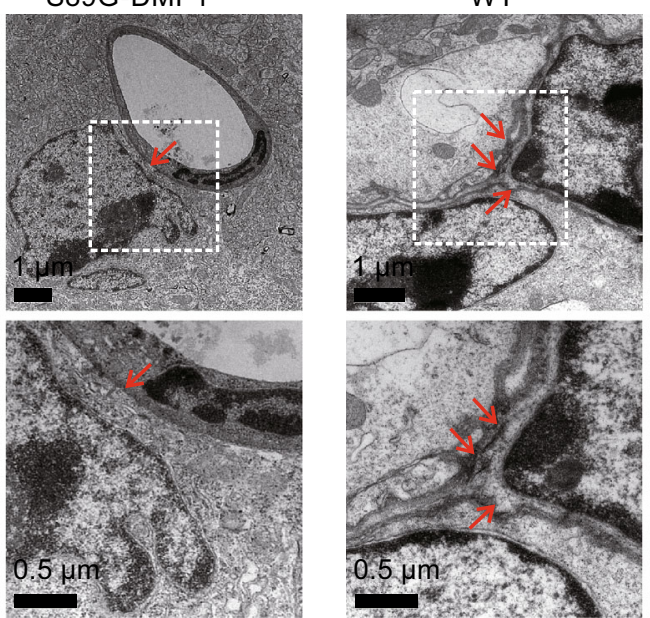

S89G-DMP1
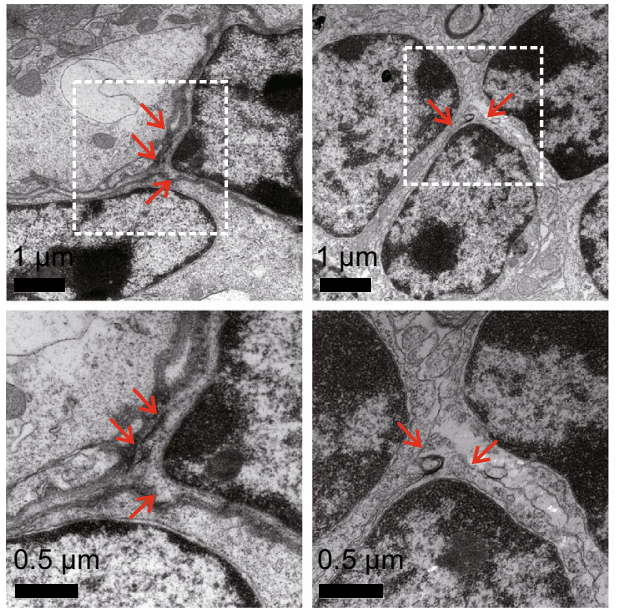

D
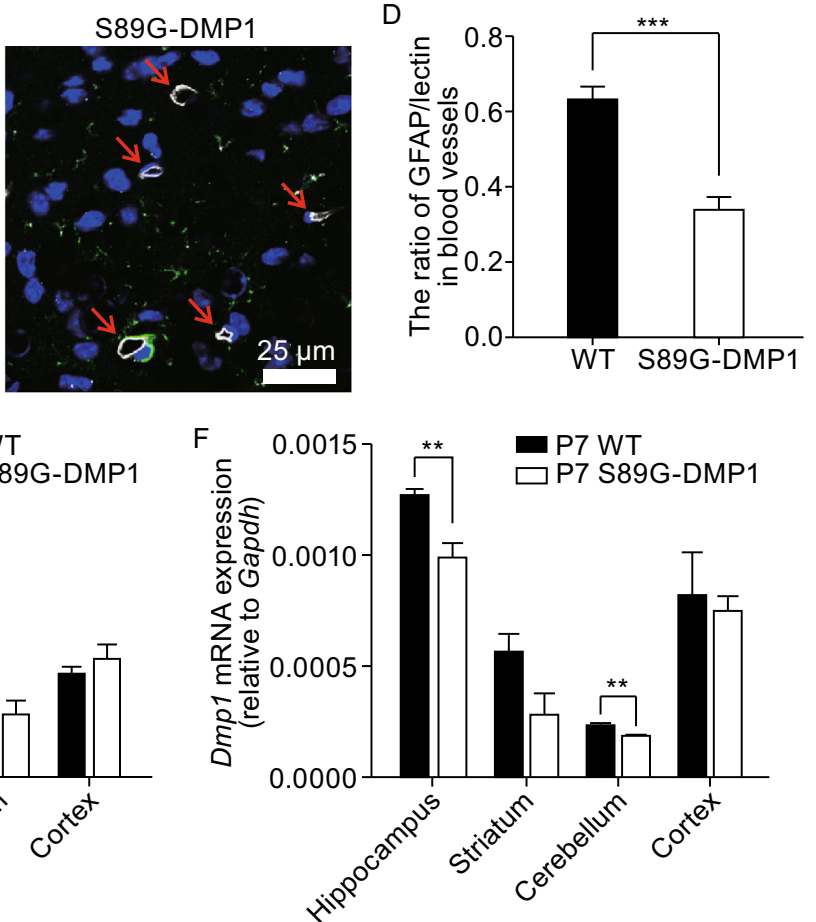

Figure 2. S89G-DMP1 inhibits astrocytes to locate to and wrap around blood vessels. (A) Transmission electron microscopeshowed loosened cell adhesion between astrocytes and vascular endothelial cells in the retrosplenial granular cortex (RSG) of S89G-DMP1 mice; and between astrocytes themselves (B); (C) Representative images of GFAP/lectin in the RSG, indicative of attenuated targeting of astrocytes to blood vessels in S89G mice; (D) Quantification plot for (C). ${ }^{* *}, P<0.001$. At least 23 random captures from7 mice per genotype were quantified. (E) Dmp1 mRNA decreased in different brain regions at embryonic Day 18.5 ( $P \leq 0.05$ vs. WT) and (F) at postnatal Day 7 ( $P \leq 0.01$ vs. WT). $n=3-4$ mice per genotype.

\section{OPEN ACCESS}

This article is distributed under the terms of the Creative Commons Attribution 4.0 International License (http://creativecommons.org/ licenses/by/4.0/), which permits unrestricted use, distribution, and reproduction in any medium, provided you give appropriate credit to the original author(s) and the source, provide a link to the Creative Commons license, and indicate if changes were made. 